\title{
On the Complexity of Partial Order Properties
}

\author{
Stefan Felsner* Dorothea Wagner ${ }^{\dagger}$
}

\begin{abstract}
The recognition complexity of ordered set properties is considered, i.e. how many questions have to be asked to decide if an unknown ordered set has a prescribed property. We prove a lower bound of $\Omega\left(n^{2}\right)$ for properties that are characterized by forbidden substructures of fixed size. For the properties being connected, and having exactly $k$ comparable pairs we show that the recognition complexity is $\left(\begin{array}{c}n \\ 2\end{array}\right)$; the complexity of interval orders is exactly $\left(\begin{array}{l}n \\ 2\end{array}\right)-1$. Non-trivial upper bounds are given for being a lattice, containing a chain of length $k \geq 3$ and having width $k$.
\end{abstract}

\section{Introduction and Overview}

A well studied recognition problem on sets arising in the context of representing sets in computer storage is defined by the following game. Given a finite set $S$ and a property $\mathcal{P}$ of subsets of $S$, i.e. $\mathcal{P} \subseteq 2^{S}$ (the powerset of $S$ ), a player $A$ wants to know if an unknown set $X \subseteq S$ is in $\mathcal{P}$ by asking questions about elements of $S$. For his questions A chooses some $x \in S$ and asks "Is $x \in X$ ?", player $B$ answers "yes" or "no". The aim of $A$ is to minimize the number of questions, while $B$ tries to force $A$ to ask as many questions as possible. In any case, the game ends up with sets $X$ and $Z$ such that either all $Y$ containing $X$ and not containing an element of $Z$, i.e $X \subseteq Y \subseteq S \backslash Z$, are in $\mathcal{P}$ or all such $Y$ are not in $\mathcal{P}$.

The number of queries necessary to finish the game if both players play optimally is called the recognition complexity of $\mathcal{P}$. A property is called elusive, if $B$ can force $A$ to ask all possible $|S|$ questions. If $\mathcal{P}$ is considered as a Boolean function, the complexity of $\mathcal{P}$ is a lower bound for the time any algorithm recognizing $\mathcal{P}$ must take in the worst case on any model of sequential machine [10].

A famous and well studied special case of this game is, when $S$ is regarded as the set of possible edges of a graph on $n$ vertices, i.e. $\mathcal{P}$ is a property of graphs [2], [6], [7], [8], [9]. The relation between this concept of recognition complexity of graph properties and the computer representation of graphs is discussed in [11]. See also [1] for more information and references on recognition complexity.

\footnotetext{
*Institut für Informatik, Fachbereich Mathematik, Freie Universität Berlin, Takustraße 9, 14195 Berlin, Germany

${ }^{\dagger}$ Fachbereich Mathematik, Technische Universität Berlin, Straße des 17. Juni 136, 10623 Berlin, Germany
} 
In [5] Faigle and Turán suggest to play the game on properties of partial orders. Here player $A$ asks for the comparability status of two elements $a$ and $b$, and $B$ answers " $a<b ", " a>b$ " or " $a$ and $b$ are incomparable."

Considering a property $\mathcal{P}$ of partial orders with $n$ elements, $\mathcal{P}$ is elusive if $B$ can force $A$ to ask all possible $\left(\begin{array}{l}n \\ 2\end{array}\right)$ questions. Obviously, the game for properties of partial orders does not fit into the concept of set properties discussed before, since there are three possible answers instead of two. Moreover, the transitivity of partial orders may lead to situations, where player $A$ knows the comparability status of two elements without asking it - independently from the considered property. While in the case of graph properties it seems that nearly all properties are elusive or at least of complexity $\Omega\left(n^{2}\right)$, there exist many "easy" properties of partial orders. E.g. the recognition problem of being a linear order is just the sorting problem and thus has complexity $O(n \log n)$.

In this paper we study the recognition complexity of several properties of partial orders. First we describe situations that induce the comparability status of an unasked pair of elements independently from the considered property. For properties that are characterized by forbidden substructures of fixed size we prove a lower bound of $\Omega\left(n^{2}\right)$ for the recognition complexity. In section 3 we prove elusiveness for connectedness and having exactly $k$ comparable pairs, for fixed $k$. Non-trivial upper bounds are given in section 4 for being a lattice, containing a chain of length $k$, for $k \geq 3$ and having width $k$, for $k$ fixed, thus proving that these properties are not elusive. For the class of interval orders we prove that $\left(\begin{array}{l}n \\ 2\end{array}\right)-1$ is the exact value of its recognition complexity.

\section{Some general observations}

We first introduce some basic notations. A partial order $P=(V,<)$ consists of a finite ground set $V$ and the order relation $<$, incomparability is denoted by $\|$. An element $b$ covers $a$ (denoted $a \prec b$ ) if $a<b$ and there is no $c \in V$ with $a<c<b$. Throughout this paper we illustrate partial orders by their Hasse diagram. The vertices of the Hasse diagram are the elements of $V$ and $b$ covers $a$ in $P$ iff $a$ and $b$ are connected by an edge going from $a$ up to $b$. A partial order property $\mathcal{P}$ is a set of partial orders over the same ground set closed under isomorphism.

Consider the game introduced in section 1 for a partial order property $\mathcal{P}$ over a $n$ element ground set $V$. The state of the game after $q \leq\left(\begin{array}{c}n \\ 2\end{array}\right)$ questions can be interpreted as a triple $((C,<), I, N)$, where $(C, I, N)$ is a partition of the set of all two-element subsets of $V$. The pairs in $C$ are those which have been given comparable in one of the $q$ steps and $<$ is the corresponding order relation. $I$ is the set of pairs given incomparable and $N$ is the set of pairs not yet asked for.

We call a triple $((C,<), I, N)$ legal if there exists a partial order $P=\left(V,<_{P}\right)$ compatible with the triple, i.e. satisfying

1. If $\{a, b\} \in C$ and $a<b$ then $a<_{P} b$.

2. If $\{a, b\} \in I$ then $a \| b$ in $P$. 
An algorithm for player $\mathrm{A}$ is a mapping $\varphi$ assigning to each legal triple $((C,<), I, N)$ a pair $\{a, b\} \in N$, i.e. $\varphi$ prescribes the next question " $a: b$ " at state $((C,<), I, N)$.

A strategy for player $\mathrm{B}$ is a mapping $\psi$ which assigns to a given legal triple $((C,<$ ), $I, N)$ and $\{a, b\} \in N$ a new legal triple which is one of the following two

$$
((C,<), I \cup\{a, b\}, N \backslash\{a, b\}) \quad, \quad((C \cup\{a, b\},<), I, N \backslash\{a, b\}) .
$$

A game is finished at state $((C,<), I, N)$ if either all partial orders $P$ compatible with the triple are in $\mathcal{P}$, or for all of them $P \notin \mathcal{P}$ holds.

The complexity of a property $\mathcal{P}$ for a fixed algorithm $\varphi$ and a fixed strategy $\psi$ is the minimum number of questions needed to finish a game if player A uses $\varphi$ and player $B$ uses $\psi$, i.e.

$$
\mathbf{C}(\mathcal{P} ; \varphi, \psi)=\min \{q \mid \text { game finishes at state }((C,<), I, N,) \text { with }|C \cup I|=q\} .
$$

The complexity of a property $\mathcal{P}$ is the minimum number of questions needed to finish a game if both $\mathrm{A}$ and $\mathrm{B}$ play optimally, i.e.

$$
\mathbf{C}(\mathcal{P})=\min _{\varphi} \max _{\psi} \mathbf{C}(\mathcal{P} ; \varphi, \psi) .
$$

For a legal triple $((C,<), I, N)$ with $|C \cup I|=q$, the number of pairs of elements whose comparability status is known may be more than $q$. We now give situations, where the comparability status of a pair $\{a, b\} \in N$ is induced by the comparability status of some other pairs independent from the partial order property under consideration.

Situation 1 If there exist elements $a_{1}, a_{2}, a_{3}$ with $a_{1}<a_{2}$ and $a_{2}<a_{3}$ then by transitivity $a_{1}<a_{3}$ holds.

Situation 2 If there exist elements $a_{1}, a_{2}$ and $b_{1}, b_{2}$ with $a_{1}<a_{2}, b_{1}<b_{2}, a_{1} \| b_{2}$ and $a_{2} \| b_{1}$ then both of $a_{1} \| b_{1}$ and $a_{2} \| b_{2}$ hold.

Proof: With each of the 4 possible comparabilities $a_{2}<b_{2}, b_{2}<a_{2}, a_{1}<b_{1}$ and $b_{1}<a_{1}$ we would introduce as transitive edge either $a_{1}<b_{2}$ or $b_{1}<a_{2}$ contradicting the incomparability of this pair. (See figure 1a).

(We always illustrate partial orders by their Hasse diagram with solid lines, incomparabilities are denoted by dashed edges, and dotted edges denote an unknown comparability status)

Situation 3 Consider a state $((C,<), I, N)$ of a game where there exists a 5 -chain $a_{1}<a_{2}<a_{3}<a_{4}<a_{5}$ and an element $b \notin\left\{a_{1}, \ldots, a_{5}\right\}$ with $\left\{b, a_{i}\right\} \in N$. Then player A can deduce the comparability status of all five pairs $\left\{b, a_{i}\right\}, 1 \leq i \leq 5$ by asking only four questions.

Proof: Player A asks for the comparability status of the pairs $b: a_{2}$ and $b: a_{4}$. If one of these pairs is comparable we gain a transitive edge. In case both pairs are given incomparable A concludes $b \| a_{3}$. (See figure 1b). 


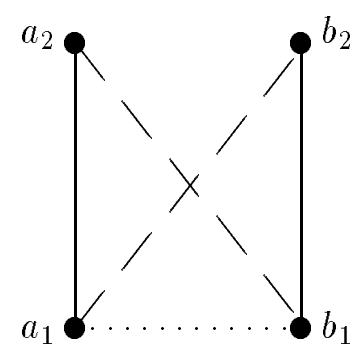

a)

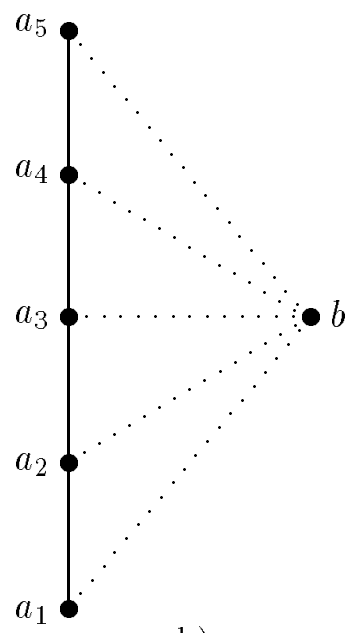

b)

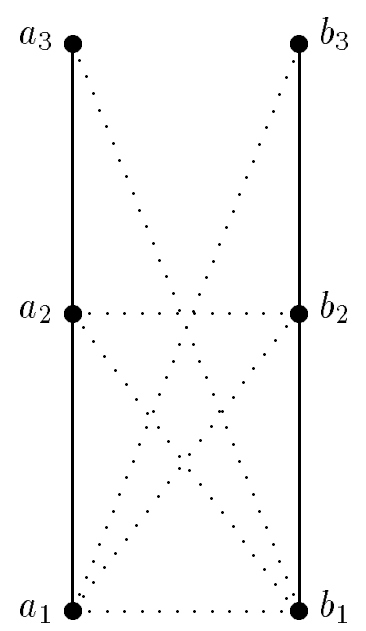

c)

Figure 1: Three standard situations.

Situation 4 Consider a state $((C,<), I, N)$ of a game where there exist two 3-chains $a_{1}<a_{2}<a_{3}$ and $b_{1}<b_{2}<b_{3}$ and all the pairs $\left\{a_{i}, b_{j}\right\}$ are in $N$. Then player A can deduce the comparability status of all six pairs $a_{i}: b_{j}$ by asking only five questions.

Proof: Player A asks for the comparability status of $a_{1}: b_{2}$ and $b_{1}: a_{2}$. If both $a_{1} \| b_{2}$ and $b_{1} \| a_{2}$, then situation 2 applies, i.e. $a_{1} \| b_{1}$ and $a_{2} \| b_{2}$. Otherwise, i.e. if at least one of these pairs is given comparable, A gains a transitive edge. (See figure 1c).

A partial order $P_{0}=\left(V_{0},<_{0}\right)$ is a suborder of $P=(V,<)$ if $V_{0} \subseteq V$ and $a<_{0} b$ iff $a<b$ for all $a, b \in V_{0}$. (In this case, we do not distinguish between $<_{0}$ and $<$.)

Theorem 1 Let $\mathcal{P}$ be a partial order property over a n-element set $V$ such that

1. $\mathcal{P}$ contains the n-element antichain;

2. there exists a partial order $P_{0}=\left(V_{0},<_{0}\right)$ of fixed size $k$, i.e. $\left|V_{0}\right|=k<n$, such that each partial order $P$ that contains $P_{0}$ as a suborder is not in $\mathcal{P}$.

Then $\mathcal{P}$ has complexity $\Omega\left(n^{2}\right)$.

Proof: Player B can make use of the following 'greedy strategy'. As long as there is a subset $X \subseteq V$ with $|X|=k$ and $\left(\begin{array}{c}X \\ 2\end{array}\right) \subseteq N$ (where $\left(\begin{array}{l}X \\ 2\end{array}\right)=\{\{x, y\} \mid x, y \in X\}$ ) the answer to the question $a: b$ is $a \| b$. The $n$-antichain and the order $P_{0}$ on $X$ together with $n-k$ independent elements are compatible orders, one in $\mathcal{P}$ the other not in $\mathcal{P}$.

Therefore player A has to ask at least one question from each $k$-element subset $X \subseteq V$. There exist $\left(\begin{array}{l}n \\ k\end{array}\right)$ different subsets of size $k$. On the other hand, a given pair of 
elements is contained in $\left(\begin{array}{c}n-2 \\ k-2\end{array}\right)$ of these sets. So, the number of questions A has to ask is at least $\frac{\left(\begin{array}{l}n \\ k\end{array}\right)}{\left(\begin{array}{l}n-2 \\ k-2\end{array}\right)}=\frac{n^{2}-1}{k^{2}-1}$, which is of $\Omega\left(n^{2}\right)$.

\section{Remark:}

1) Obviously, the complexity of a property $\mathcal{P}$ is equal to the complexity of its complement, i.e. the set of all partial orders that are not in $\mathcal{P}$ (which is a partial order property as well).

2) Theorem 1 applies to a lot of partial order properties, e. g. for being an interval order, being a lattice, having dimension at most 2 or containing a chain of length at least 3 .

\section{Elusive Properties}

Let us call a partial order connected if its Hasse diagram considered as an undirected graph is connected.

Theorem 2 The property $\mathcal{P}$ of all connected partial orders over set $V$ is elusive.

Proof: We give a strategy $\psi$ for player B such that $\mathbf{C}(\mathcal{P} ; \varphi, \psi)=\left(\begin{array}{c}n \\ 2\end{array}\right)$ for all algorithms $\varphi$ of player A.

Let the first question be $a: b$, then $\mathrm{B}$ answers $a<b$. For further questions $a: b$ he answers $a \| b$, except in case $\{a, b\}$ is the last possible edge between one of the elements, say $a$, that is not comparable to another element, and an element $b$ comparable to some other element. Then the comparability is given according to the comparability status of $b$, such that $b$ remains a minimal element or a maximal element. More precisely, for a legal $((C,<), I, N)$ let $M=\bigcup_{\{x, y\} \in C}\{x, y\}$ then for $\{a, b\} \in N$ the answer of B is:

$a<b$ if $a \notin M, b \in M$ and for all $x \in M \backslash\{b\}$ we have $\{x, a\} \in I$, and $b>c$ for some $c \in M$;

$b<a$ if $a \notin M, b \in M$ and for all $x \in M \backslash\{b\}$ we have $\{x, a\} \in I$, and $b<c$ for some $c \in M$;

$a \| b$ else.

The strategy $\psi$ obviously preserves the invariants:

(1) The partial order induced by $(C,<)$ over $M$ is connected, and $\left(\begin{array}{c}M \\ 2\end{array}\right) \subseteq C \cup I$. 
(2) All $x \in M$ are either minimal or maximal with respect to $(C,<)$.

(3) For each $x \in V \backslash M$ there is a $y \in M$ such that $\{x, y\} \in N$.

Applying $\psi$, the game ends with a legal triple $((C,<), I, N)$. If $|C \cup I|<\left(\begin{array}{c}n \\ 2\end{array}\right)$, then the partial orders compatible with $((C,<), I, N)$ would all be connected or all be disconnected. But invariants 1 and 2 contradict the assumption that all compatible partial orders are connected, while invariant 3 contradicts the case that they all are not connected.

Theorem 3 The property $\mathcal{P}$ of all partial orders containing exactly $k$ comparable pairs over $V$ with $|V|=n$ is elusive if $k=n_{1} \cdot n_{2}$ with $n_{1}+n_{2} \leq n$.

Proof: A strategy $\psi$ for player B such that $\mathbf{C}(\mathcal{P} ; \varphi, \psi)=\left(\begin{array}{l}n \\ 2\end{array}\right)$ for all algorithms $\varphi$ is to construct a "complete height 1 order" with exactly $k$ edges. Let $(X, Y, Z)$ be a partition of $V$ with $|X|=n_{1}$ and $|Y|=n_{2}$, the order $P_{0}$ on $V$ is defined by $x<y$ iff $x \in X$ and $y \in Y$. The number of comparabilities of $P_{0}$ is $k$.

Given a question $a: b$ the answer of player $B$ is the comparability stat us of the pair $\{a, b\}$ in $P_{0}$. The length of the game then is $\left(\begin{array}{c}n \\ 2\end{array}\right)$ since even the last unasked pair may change the number of comparabilities of the final order $P$. Let $\{a, b\}$ be the last unasked pair. If $a$ and $b$ are comparable in $P_{0}$ then $\mathrm{B}$ may give $a \| b$ and the game ends with an order with $k-1$ comparabilities. If $a \| b$ in $P_{0}$ then $\mathrm{B}$ may give $a<b$ if $a \in X$ and $b<a$ otherwise, the resulting order then has $k+1$ comparabilities.

\section{Remark:}

1) The proof shows that the recognition complexity of the order $P_{0}$ is $\left(\begin{array}{l}n \\ 2\end{array}\right)$ while in [5] height 1 orders are presented which have recognition complexity of $O(n \log n)$.

2) Let $f(k)$ be the complexity of having exactly $k$ comparable pairs. By the theorem $f(k)=\left(\begin{array}{l}n \\ 2\end{array}\right)$ for many values $k \leq \frac{n^{2}}{4}$, on the other hand $f(k) \in \Theta(n \log n)$ for $k \geq \frac{n^{2}}{2}-c n$ for $c$ constant. It would be interesting to know the value of $f(k)$ for some $k$ in between.

\section{Upper Bounds}

In this section we give upper bounds for the complexity of several partial order properties. Consider a partial order $P=(V,<)$, two elements $a, b \in V$ have the minimum $x \in V$, denoted $x=\min \{a, b\}$ if $x \leq a$ and $x \leq b$, and $z \leq a$ and $z \leq b$ implies $z \leq x$. 


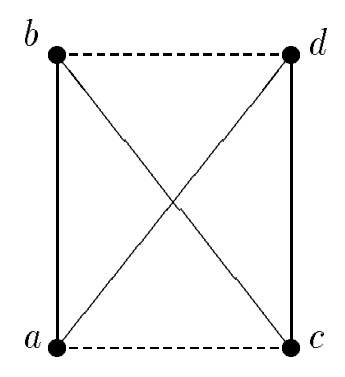

Figure 2: Forbidden suborder for lattices.

The maximum is defined analogously. $P=(V,<)$ is a lattice iff $\min \{a, b\}$ and $\max \{a, b\}$ exist for all $a, b \in V$.

Theorem 4 Let $\mathcal{P}$ be the set of all lattices over $V,|V|>3$, then $\mathbf{C}(\mathcal{P})<\left(\begin{array}{l}n \\ 2\end{array}\right)$.

Proof: In the following we use the fact that $L=(V,<)$ is a lattice iff it does not contain four elements $a, b, c, d$ with $a \prec b, a \prec d, c \prec b, c \prec d, a \| c$ and $b \| d$ (see figure 2), and it contains a unique minimum and a unique maximum, i.e. an element $x \in V$ such that $x \leq y$ respectively $y \leq x$ for all $y \in V$. (Denote the minimum resp. maximum of $L$ by $\min (L)$ resp. $\max (L)$.)

An algorithm $\varphi$ for player $\mathrm{A}$ with $\mathbf{C}(\mathcal{P} ; \varphi, \psi)<\left(\begin{array}{c}n \\ 2\end{array}\right)$ for all strategies $\psi$ is first to ask all $\left(\begin{array}{c}n-1 \\ 2\end{array}\right)$ questions over $V \backslash\{x\}$ for a fixed $x \in V$. The state of the game after these $\left(\begin{array}{c}n-1 \\ 2\end{array}\right)$ questions is a legal triple $((C,<), I, N)$ and $N=\{\{x, y\} \mid y \in V\}$.

Case 1 The partial order induced by $((C,<), I)$ is not a lattice. The 'defect' of $((C,<), I)$ relative to lattices has to be so small that adding $x$ in the right way leads to a lattice. The possible situations then are

1.1 The unique minimum or maximum is missing. Then, w.l.o.g. let $((C,<), I)$ induce a partial order containing no minimum. It must contain a maximum $y$ and all lattices compatible with $((C,<), I, N)$ contain $x$ as its minimum. So A asks $a: x$ for an arbitrary $a \in V \backslash\{x, y\}$. Player $\mathrm{B}$ has to answer $x<a$, else there is no compatible partial order that is a lattice, but with $x<a$ and $x<y$ the transitive edge $a<y$ is given.

1.2 The partial order induced by $((C,<), I)$ contains a forbidden substructure on elements $a, b, c, d$. In this case A asks $b: x$ and $d: x$. Then $\mathrm{B}$ either gives a transitive edge between $x$ and the minimum or the maximum, or B answers $b \| x$ and $d \| x$, which implies that there exists no compatible partial order which is a lattice.

Case 2 The partial order induced by $((C,<), I)$ is a lattice.

2.1 If the lattice contains a 5-chain, then situation 3 from section 2 applies. 
2.2 If the lattice has height 3 , i.e. there is a 4 chain $\min <a_{1}<b_{1}<\max$, then the nonextremal elements are partitioned into $a_{1}, \ldots, a_{k}$, those covering the minimum, and the remaining elements $b_{1}, \ldots, b_{l}$. Note that all the $b_{i}$ are covered by the maximum. A first asks $x: a_{i}$ for $2 \leq i \leq k$. If $x$ is comparable with at least one of the $a_{i}$ then this comparability induces a transitive edge. Hence we assume that $\mathrm{B}$ always answers $x \| a_{i}$. Now, A asks $x: b_{j}$ for $1 \leq j \leq l$. Again, the comparability of $x$ with one of the $b_{j}$ would induce a transitive edge. Assume $x \| b_{j}$ for $1 \leq j \leq l$. The next two questions are $x: \min$ and $x: \max$. To guarantee that there exists a compatible partial order that is a lattice, $\mathrm{B}$ has to answer $x<\max$ and $\min <x$. But now the comparability status of $x$ and $a_{1}$ may be chosen arbitrarily, since all partial orders compatible with that state of the game do not contain the forbidden substructure, i.e. are lattices.

2.3 If the lattice has height $2, \mathrm{~A}$ first asks $x: \min$ and $x: \max$. If the answers of $\mathrm{B}$ are $\min \nless x$ or $x \nless \max , \mathrm{B}$ either gives a transitive edge or there exist no compatible partial orders which are lattices.

Otherwise, if $\min <x<\max$, then the comparability status of $x$ and all other elements of $V$ may be chosen arbitrarily, since there exists no compatible partial order that contains a forbidden substructure, i.e. all compatible partial orders are lattices.

Theorem 5 The property $\mathcal{P}$ of all partial orders over set $V$ with $|V|=n \geq 4$, that contain a k-chain, $k \geq 4$ has complexity $\mathbf{C}(\mathcal{P})<\left(\begin{array}{l}n \\ 2\end{array}\right)$.

Proof: A asks all possible questions over $V \backslash\{x\}$ for fixed $x$. To guarantee that for the state of the game after these $\left(\begin{array}{c}n-1 \\ 2\end{array}\right)$ questions there exists a compatible partial order containing a chain of length $k, \mathrm{~B}$ has to construct a chain of length $k-1$, say $a_{1}<a_{2}<\ldots<a_{k-1}$. Now A asks $x: a_{2}$. If $\mathrm{B}$ answers $x \| a_{2}$, then the comparability status of $x: a_{1}$ and $x: a_{i}, 2<i \leq k-1$, is not essential for $\mathcal{P}$. Otherwise, if $\mathrm{B}$ answers $x<a_{2}$ or $a_{2}<x$ there is an induced transitive edge.

This argument does not apply to the case $k=3$.

Theorem 6 The property $\mathcal{P}$ of all partial orders over set $V,|V|=n \geq 5$, that contain a 3-chain has complexity $\mathbf{C}(\mathcal{P})<\left(\begin{array}{l}n \\ 2\end{array}\right)$.

Proof: We use the following two facts.

Fact 1 If for a strategy $\psi$ there exists a state $((C,<), I, N)$ with $\{a, b\},\{c, d\} \in C$, $a<b, c<d$ and $\{a, d\} \in N,\{b, d\},\{a, c\} \in N \cup I$ then there exists an algorithm $\varphi$ such that $\mathbf{C}(\mathcal{P} ; \varphi, \psi)<\left(\begin{array}{l}n \\ 2\end{array}\right)$. 


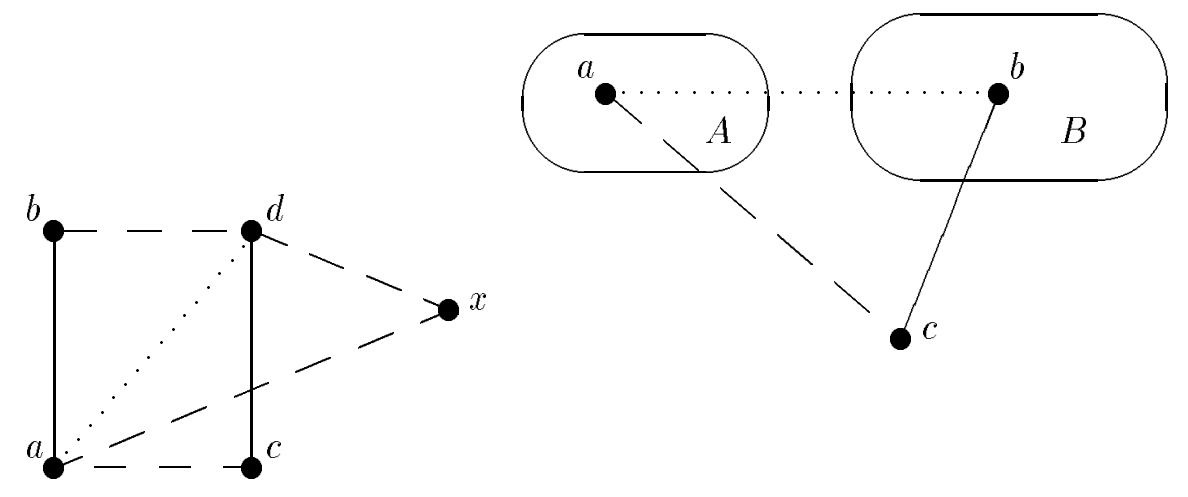

Figure 3: Illustrations for the facts.

A may obtain $\{a, c\} \in I$ and $\{d, b\} \in I$, otherwise there would be a 3 -chain. Now A asks all remaining questions $\{a, x\}, x \neq d$ and all questions $\{d, x\}, x \neq a$. If $\{a, x\} \in C$ then $a<x$, otherwise we would have a 3 -chain, symmetrically $\{d, x\} \in C$ implies $x<d$. From this we conclude that for all $x \neq a, d$ either $\{a, x\} \in I$ or $\{d, x\} \in I$, but now the comparability status of $a: d$ is not essential for $\mathcal{P}$, since this pair can not contribute to a 3 -chain in a compatible order. (See figure 3 ).

Fact 2 If for a strategy $\psi$ there exists a state $((C,<), I, N)$ such that

i) $\{c, x\} \in C \cup I$ for a fixed $c \in V$ and all $x \in V \backslash\{c\}$,

ii) there are $a, b \in V$ with $\{a, c\} \in I,\{b, c\} \in C$,

iii) for all $a, b \in V$ with $\{a, c\} \in I,\{b, c\} \in C$, we have $\{a, b\} \in N$

then there exists an algorithm $\varphi$ such that $\mathbf{C}(\mathcal{P} ; \varphi, \psi)<\left(\begin{array}{c}n \\ 2\end{array}\right)$.

W.1.o.g. $c<b$. Let $A=\left\{a_{i} \in V \backslash\{c\}:\left\{c, a_{i}\right\} \in I\right\}$ and $B=\left\{b_{j} \in V \backslash\{c\}:\left\{c, b_{j}\right\} \in\right.$ $C\}$, then $c<b_{j}$ for all $b_{j} \in B$ since otherwise there is a 3-chain. We next ask for all the remaining pairs $\left\{a_{i}, a_{j}\right\}$ and $\left\{b_{i}, b_{j}\right\}$. The pairs $\left\{a_{i}, a_{j}\right\}$ are given incomparable, otherwise fact 1 applies. Moreover, all pairs $\left\{b_{i}, b_{j}\right\}$ are given incomparable to avoid a 3 -chain. But then there exists no compatible partial order in $\mathcal{P}$, i.e. the comparability status of all $a_{i}: b_{j}$ for $a_{i} \in A$ and $b_{i} \in B$ is not essential for property $\mathcal{P}$ since $b_{j}$ cannot cause a 3 -chain. (See figure 3 ).

An algorithm $\varphi$ with $\mathbf{C}(\mathcal{P} ; \varphi, \psi)<\left(\begin{array}{c}n \\ 2\end{array}\right)$ for all strategies $\psi$ is the following. Let $V=\left\{x_{1}, \ldots, x_{n}\right\}$. First, $\mathrm{A}$ asks $x_{1}: x_{2}$. If the answer is $x_{1} \| x_{2}$ then $\mathrm{A}$ asks $x_{1}: x_{i}$ for $2<i \leq n-1$. B answers $x_{1} \| x_{i}$, otherwise fact 2 applies. But then the comparability status of $x_{1}: x_{n}$ is not essential, since neither $x_{1}<x_{n}$ nor $x_{n}<x_{1}$ can contribute to a 3-chain.

So, assume B answers w.l.o.g. $x_{1}<x_{2}$. Now, A asks $x_{1}: x_{i}$, for $2<i \leq n-2$. Because of fact 2 , respectively to avoid a chain of length three, $\mathrm{B}$ always answers $x_{1}<x_{i}$. 
With the following questions, A can force a situation where the comparability status of $x_{n-1}: x_{n}$ is not essential for $\mathcal{P}$.

First question A asks $x_{2}: x_{n}$.

To avoid a 3 -chain, respectively because of fact 1 with $a=x_{n}, b=x_{2}, c=x_{1}$, $d=x_{3}$, B answers $x_{2} \| x_{n}$.

Second set of questions A asks all $x_{i}: x_{n}$ for $2<i \leq n-2$.

To avoid a chain of length $3, \mathrm{~B}$ will in no case answer $x_{i}<x_{n}$. Assume $\mathrm{B}$ answers $x_{i} \| x_{n}$ for all $i, 2<i \leq n-2$. Then $\mathrm{A}$ asks $x_{n-1}: x_{n}$, and if $\mathrm{B}$ answers $x_{n-1} \| x_{n}$ $x_{1}: x_{n}$ is not essential for $\mathcal{P}$. But a comparibility between $x_{n}$ and $x_{n-1}$ induces fact 1. Thus for at least one $x_{i}, 2<i \leq n-2 \mathrm{~B}$ will answer $x_{n}<x_{i}$. W.l.o.g. let $x_{n}<x_{3}$.

Third set of questions A asks $x_{1}: x_{n-1}$.

The answer $x_{n-1}<x_{1}$ induces a chain of length three.

If $x_{1} \| x_{n-1}$, then $\mathrm{A}$ asks all questions $x_{i}: x_{n-1}$ for $2 \leq i<n$, and either gets a comparability which induces a chain of length three or fact 1 , or $x_{i} \| x_{n-1}$ for all $i$, and thus $x_{n-1}: x_{n}$ is not essential for $\mathcal{P}$.

So let $\mathrm{B}$ answer $x_{1}<x_{n-1}$.

Fourth set of questions $\mathrm{A}$ asks $x_{i}: x_{n}$, for all remaining $x_{i}, 1 \leq i<n-1$, strating with $x_{1}: x_{n}$.

To avoid 3-chains B has to answer $x_{1} \| x_{n}$. For all other $x_{i}$ the answers are $x_{i} \| x_{n}$ or $x_{n}<x_{i}$. At answers $x_{n}<x_{i}$ let the question $x_{i}: x_{n-1}$ follow, this has to be answered with $x_{i} \| x_{n-1}$ to avoid a 3 -chain.

But now, the comparability status of $x_{n-1}: x_{n}$ is not essential for $\mathcal{P}$, since each $x_{i}$, $i \neq n, n-1$ is incomparable to one of $x_{n-1}$ and $x_{n}$.

Theorem 6 is a kind of indicator that elusive partial order properties must be of low height. We now consider the width of partial orders, i.e. the maximal size of an antichain.

Theorem 7 Let $\mathcal{P}$ be the property of all partial orders of width $k$ over $V$, for a fixed $k$, then $\mathbf{C}(\mathcal{P}) \leq 2 k n \log n$.

Proof: The algorithm $\varphi$ with $\mathbf{C}(\mathcal{P} ; \varphi, \psi) \leq 2 k n \log n$ is based on sorting. Let the ground set be indexed, i.e $V=\left\{x_{1}, \ldots, x_{n}\right\}$, then player A determines one after another the order on $\left\{x_{1}, \ldots, x_{i}\right\}$ for $1 \leq i \leq n$. Consider $P_{i}=\left(\left\{x_{1}, \ldots, x_{i}\right\},<\right)$, if the width of $P_{i}$ is more than $k$, then all compatible orders have this property and the game is over. 
Therefore we assume the width of $P_{i}$ to be at most $k$ and, by the theorem of Dilworth [3] $P_{i}$ can be partitioned into $k$ chains $H_{i}^{1}, \ldots, H_{i}^{k}$.

Let $H_{i}^{j}$ be a chain of the chain partition of $P_{i}$, say $H_{i}^{j}=c_{1}<c_{2}<\ldots<c_{l}$. A determines the comparability status of $\left\{x_{i+1}, c_{j}\right\}$ for $1 \leq j \leq l$ using binary search. First $A$ asks for $x_{i+1}: c_{\left\lceil\frac{l}{2}\right\rceil}$. If $x_{i+1}<c_{\left\lceil\frac{l}{2}\right\rceil}$ (resp. $\left.x_{i+1}>c_{\left\lceil\frac{l}{2}\right\rceil}\right)$ then A recursively determines the comparability status of $x_{i+1}$ with the elements of the remaining 'halfchain' $\left\{c_{j} \mid 1 \leq j<\left\lceil\frac{l}{2}\right\rceil\right\}$ (resp. $\left\lceil\frac{l}{2}\right\rceil<j \leq l$ ).

If $x_{i+1} \| c_{\left\lceil\frac{l}{2}\right\rceil}$, then $l_{1}<\left\lceil\frac{l}{2}\right\rceil<l_{2}$ holds, where $l_{1}:=\max \left\{j \mid j=0\right.$ or $\left.c_{j}<x_{i+1}\right\}$ and $l_{2}:=\min \left\{j \mid j=l+1\right.$ or $\left.x_{i+1}<c_{j}\right\}$. Now, A recursively applies bitonic sort to both half-chains to determine $l_{1}$ and $l_{2}$. The comparability status of $x_{i+1}$ with all elements of $H_{i}^{j}$ can thus be determined with $2 \log l$ questions. The comparability status of all pairs from $\left\{x_{1}, \ldots, x_{i}\right\}$ is known after at most $2 k \log n$ queries. Adding the $n$ elements one by one we obtain the overall complexity of $2 k n \log n$.

Remark: Algorithm $\varphi$ not only decides if an unknown partial order has width at most $k$, but also if it is isomorphic to a fixed partial order $P_{0}$ of width $k$. Thus theorem 7 improves the upper bound given in [5] (which is $2 k n \log n+3 k n$ ) for the $P_{0}$-recognition problem.

A partial order $P=(V,<)$ is an interval order iff there exists a collection $\left(I_{x}\right)_{x \in V}$ of intervals on the real line, such that $x<y$ iff $I_{x}$ lies entirely to the left of $I_{y}$. The characterization theorem of Fishburn says: $P$ is an interval order iff $P$ does not contain a suborder $\mathbf{2 + 2}$, where $\mathbf{2 + 2}=(\{a, b, c, d\},<)$ with $a<b, c<d$ and no further comparabilities [4].

Theorem 8 The recognition complexity of the class $\mathcal{P}$ of interval orders is $\mathbf{C}(\mathcal{P})=$ $\left(\begin{array}{l}n \\ 2\end{array}\right)-1$.

Proof: We first prove $\mathbf{C}(\mathcal{P}) \leq\left(\begin{array}{c}n \\ 2\end{array}\right)-1$. This is done by describing an algorithm $\varphi$, such that $\mathbf{C}(\mathcal{P} ; \varphi, \psi) \leq\left(\begin{array}{c}n \\ 2\end{array}\right)-1$ for all strategies $\psi$. Let $V:=\left\{x_{1}, \ldots, x_{n}\right\}$, A takes the elements by increasing index and asks for their comparability status to all elements with higher index, until B gives the first comparability.

In case $x_{i} \| x_{j}$ for $i \leq n-3$ and all $j$, every compatible partial order is an interval order and A gains 3 questions. So, let $\left\{x_{k}, x_{k+l}\right\}$ be the first comparable pair, and $x_{k}<x_{k+l}$ (the other case is dual). Now, algorithm $\varphi$ uses the fact that an interval order can not contain a $\mathbf{2}+\mathbf{2}$ as suborder, and situation 2 of section 2, i.e. that in any four elements $\{a, b, c, d\} \subseteq V$, with $a<b$ and $c<d$, A only has to know the comparability status of the diagonals $\{a, d\}$ and $\{c, b\}$ to decide if the four elements induce the forbidden $\mathbf{2}+\mathbf{2}$.

A then asks all pairs of elements from $V^{\prime}=\left\{x_{k}, x_{k+1}, \ldots, x_{k+l-1}, x_{k+l+1}, \ldots, x_{n}\right\}$. Let $Q$ be the resulting order. If $Q$ is an antichain then all compatible partial orders are 
in $\mathcal{P}$. We thus assume that at least one pair is comparable. $Q$ has to be an interval order, otherwise all compatible orders contain the $\mathbf{2}+\mathbf{2}$ of $Q$ and are not in $\mathcal{P}$. Let $z \in V^{\prime}$ be an element with maximal set of predecessors in $Q$ (interval orders always contain a $z$ such that $a<b$ implies $a<z$ ). If $z=x_{k}$ then there is a $x_{j}$ with $x_{j}<x_{k}$ and we gain the transitive edge $x_{j}<x_{k+l}$, hence $z \neq x_{k}$. Now, ask the edges $\left\{x_{k+l}, x^{\prime}\right\}$ with $x^{\prime} \in V^{\prime} \backslash\left\{z, x_{k}\right\}$. We claim: if still there is no $\mathbf{2}+\mathbf{2}$ then the edge $\left\{x_{k+l}, z\right\}$ can not cause one.

Suppose there exists a set $\left\{x_{k+l}, z, x_{i}, x_{j}\right\}, k \leq i \neq j \leq n$ that can still form a $\mathbf{2}+\mathbf{2}$. From the maximality of $z$ 's set of predecessors we deduce that $x_{j}<z$. Then we can choose $x_{i}$ such that the $\mathbf{2 + 2}$ is of the form $x_{i}<x_{k+l}$ and $x_{j}<z$. But then the diagonals have already been asked and we are able to detect the $\mathbf{2 + 2}$ without asking $\left\{x_{k+l}, z\right\}$.

To prove $\mathbf{C}(\mathcal{P}) \geq\left(\begin{array}{l}n \\ 2\end{array}\right)-1$ consider the following 'greedy-strategy' $\psi$. For all states of the game and all questions $x: y \mathrm{~B}$ answers $x \| y$ unless there is no compatible partial order containing a $\mathbf{2}+\mathbf{2}$.

Let $((C,<), I, N)$ be the first state where $C \neq \emptyset$, let $C=\{a, b\}$. Consider the graph $G_{N}$ induced by $N$, i.e. with vertex set $V(N):=\left\{x_{i}\right.$ : there is a $\left.\left\{x_{i}, x_{j}\right\} \in N\right\}$ and edge set $N$. It is easy to see that $G_{N}$ is either a star or a triangle. That is, either $N \subseteq\left\{\left\{y, x_{i}\right\}: x_{i} \in V\right\}$ for some $y \in V$, or $N=\left\{\left\{x_{i}, x_{j}\right\},\left\{x_{j}, x_{l}\right\},\left\{x_{l}, x_{i}\right\}\right\}$ for some $x_{i}, x_{j}, x_{l} \in V$.

If $G_{N}$ is a star then B can force A to ask all the remaining questions from $N$ by using $\psi$.

If $G_{N}$ is a triangle and $\{a, b\} \cap\left\{x_{i}, x_{j}, x_{l}\right\}=\emptyset$, then $\psi$ forces A to ask all remaining questions from $N$ as well.

The only case where the game finishes after $\left(\begin{array}{l}n \\ 2\end{array}\right)-1$ questions is if $G_{N}$ is a triangle, $\{a, b\} \cap\left\{x_{i}, x_{j}, x_{l}\right\}=\left\{x_{i}\right\}$ and $\mathrm{A}$ first asks $x_{j}: x_{l}$. In this case A can make use of 'situation 2' and gain one question.

\section{References}

[1] M. Aigner, Combinatorial Search, (Wiley-Teubner 1988).

[2] B. Bollobas, Extremal Graph Theory, (Academic Press 1978).

[3] R. P. Dilworth, A Decomposition Theorem for Partially Ordered Sets, Ann. of Math. Vol. 51, No. 1, (1950) 161-166.

[4] P. C. Fishburn, Interval Orders and Interval Graphs, (Wiley-Interscience Series in diskrete Mathematics, John Wiley \& Sons 1985).

[5] U. Faigle and Gy. Turán, Sorting and recognition problems for ordered sets, SIAM J. Comp. 17 (1988) 100-113. 
[6] D. J. Kleitman and D. J. Kwiatkowski, Further results on the AanderaaRosenberg conjecture, J. Comb. Theory, Ser. B 28 (1980) 85-95.

[7] J. Kahn, M. Saks and D. Sturtevant, A topological approach to evasiveness, Combinatorica \& (1984) 297-306.

[8] E. C. Milner and D. J. A. Welsh, On the computational complexity of graph theoretical properties, Proc. Fifth British Combinatorial Conference (C. St. J. A. Nash-Williams and J. Sheehan, eds.) Utilitas Math., Winnipeg (1976) 471-487.

[9] A. L. Rosenberg, On the time required to recognize properties of graphs: a problem, SIGACT News 5 (1973) 15-16.

[10] R. L. Rivest and J. Vuillemin, On recognizing graph properties from adjacency matrices, Theor. Comp. Science 3 (1976) 371-384.

[11] J. van Leeuwen, Graph Algorithms, in: Handbook of Theoretical Computer Science, Algorithms and Complexity (J. van Leeuwen ed.) (Elsevier 1990) 525-632. 